\title{
Sleep disordered breathing in childhood
}

\author{
Yasemin Gokdemir and Refika Ersu
}

CrossMark

Affiliation: Division of Pediatric Pulmonology, Marmara University, Istanbul, Turkey.

Correspondence: Yasemin Gokdemir, Division of Pediatric Pulmonology, Marmara University Hospital, Fevzi Cakmak Mah.Mimar sinan cad. No:41, Istanbul, 34890, Turkey. E-mail: yase76ayahoo.com

ABSTRACT Sleep disordered breathing (SDB) in childhood is linked with significant end-organ dysfunction across various systems, particularly with cardiovascular, neurocognitive and metabolic consequences. If we understand the pathophysiology of SDB, diagnose it promptly and treat appropriately, we may be able to prevent morbidity associated with SDB and also save health resources around the world. In this article, we highlight articles on this topic published in medical journals in the past year.

0 @ERSpublications

Prematurity, obesity and adenotonsillar hypertrophy are risk factors for sleep disordered breathing http://ow.ly/W47FU

\section{Introduction}

Paediatric obstructive sleep apnoea syndrome (OSAS) is a common health problem, and one that can result in significant morbidity. By understanding the pathophysiology of OSAS and diagnosing it in time and treating appropriately, we may be able to prevent morbidity associated with OSAS, and also save health resources around the world. Researchers in this field published studies that increased our understanding of childhood OSAS in 2014 and 2015, and this review focuses on those studies.

\section{Risk factors and pathophysiology of obstructive sleep apnoea}

Adenotonsillar hypertrophy, obesity, muscle weakness and craniofacial anomalies are the major risk factors for OSAS in children. SCHWAB et al. [1] investigated anatomic risk factors in obese adolescents with OSAS compared to obese and lean control subjects using magnetic resonance imaging. They demonstrated that increased size of the pharyngeal lymphoid tissue, rather than enlargement of the upper airway soft tissue structures, is the primary anatomic risk factor for OSAS in obese adolescents. According to the trial, adenotonsillectomy should be considered as the initial treatment for OSAS in obese adolescents.

Caffeine administered for apnoea of prematurity has beneficial short- and long-term effects on survival and neurodevelopmental disabilities. In contrast, animal studies suggested that neonatal caffeine has long-term detrimental effects on sleep and control of breathing. MARcus et al. [2] performed a multicentre international study (Caffeine for Apnea of Prematurity (CAP)) and evaluated 201 children of premature birth currently 5-12 years old. All children underwent unattended ambulatory polysomnography (PSG). Ex-preterm infants, regardless of caffeine status, were at risk for OSAS and periodic limb movements in later childhood. Therapeutic neonatal caffeine administration had no long-term effects on sleep duration or sleep apnoea during childhood. This study supported previous studies indicating prematurity as an important risk factor for childhood OSAS.

TAPIA et al. [3] aimed to determine perinatal factors associated with OSAS at school age. 197 ex-preterm (weight 500-1250 g) children aged 5-12 years that participated in the CAP study were included. They showed that OSAS is associated with chorioamnionitis and multiple gestations in ex-preterm children. Those born to older white mothers appeared to be protected. The authors speculated that the former might be due to systemic inflammation and the latter to a higher socioeconomic status (SES).

Received: Nov 022015 | Accepted after revision: Dec 152015

Conflict of interest: None declared.

Provenance: Submitted article, peer reviewed.

Copyright $\odot$ ERS 2016. ERR articles are open access and distributed under the terms of the Creative Commons Attribution Non-Commercial Licence 4.0. 


\section{Diagnosis}

PSG is considered the most accurate method for determining the presence and severity of OSAS, but is costly and has limited availability. The American Academy of Pediatrics recommends overnight, attended, in-laboratory PSG as the gold-standard tool for the diagnosis of OSAS [4]. However, in a survey of paediatric otolaryngology specialists in the USA, children presenting with clinical manifestations consistent with sleep disordered breathing (SDB) were referred for PSG "most of the time" prior to adenotonsillectomy by only $4 \%$ of survey participants [5]. Alternative diagnostic tests that are easier, cheaper and more readily acceptable are needed.

Questionnaires may be useful for the assessment of SDB in children. However, these instruments must be clear, simple, brief, reliable and easy to understand and administer. Several different questionnaires are used for diagnostic purposes in routine clinical practice. Mitchell et al. [6] analysed baseline data from 453 children from the Childhood Adenotonsillectomy (CHAT) study. Children aged 5.0-9.9 years with OSAS, who were considered candidates for adenotonsillectomy, were included in the study. Race (AfricanAmerican), obesity (body mass index (BMI) z-score >2) and Pediatric Sleep Questionnaire (PSQ) score were associated with higher levels of apnoea-hypopnoea index (AHI) and oxygen desaturation index.

BRouillette and colleagues $[7,8]$ have emphasised the clinical importance of clusters of desaturation events in nocturnal oximetry recordings for prediction of SDB severity in PSG. A nocturnal oximetry recording with a duration $>6 \mathrm{~h}$ is considered diagnostic for OSAS if it demonstrates at least three clusters of desaturation events, and at least three peripheral arterial oxygen saturation $\left(\mathrm{SpO}_{2}\right)$ drops to $<90 \%$. In Canada, oximetry has been successfully implemented as an abbreviated and low-cost testing modality for the diagnosis of OSAS in childhood [8]. KADITIs et al. [9] evaluated 25 original articles to evaluate the efficacy of nocturnal oximetry in diagnosis of SDB in children. They reported that nocturnal oximetry emerged as a valuable tool that can facilitate treatment decisions when PSG is not available.

GARDE et al. [10] performed a study to combine $\mathrm{SpO}_{2}$ characterisation and pulse rate variability, in order to identify children with SDB using the phone oximeter, a device integrating a pulse oximeter with a smartphone. They demonstrated that the phone oximeter might provide an improved portable, at-home screening tool, with the capability of monitoring patients over multiple nights. More studies are needed before this device can be used clinically.

MARCus et al. [11] evaluated 201 prematurely born children, currently aged 5-12 years. These children from the CAP study underwent unattended ambulatory PSG in the home setting. The overall satisfactory rate for unattended PSG was 98\%. This large, international study showed that comprehensive, unattended, ambulatory PSG is feasible, technically adequate and well tolerated in school-aged children when performed under research conditions.

Alonso-Álvarez et al. [12] evaluated the diagnostic reliability of home respiratory polygraphy (HRP) in children with a clinical suspicion of obstructive sleep apnoea (OSA)-hypopnoea syndrome. 60 children were studied, and $66 \%$ were diagnosed with OSAS based on a PSG-defined obstructive respiratory disturbance index $\geqslant 3$ events $\cdot \mathrm{h}^{-1}$ total sleep time. They reported that HRP recordings emerge as a potentially useful and reliable approach for the diagnosis of OSAS in children. However, more research is required for the diagnosis of mild OSAS using HRP in children.

Children with low SES have increased risk of SDB. Unfortunately, their access to subspecialty care is often limited. Boss et al. [13] reported that children with low SES experienced longer intervals from initial evaluation to PSG or adenotonsillectomy.

If we can recognise children at increased risk for OSAS using simple clinical tools, we can avoid the delay in diagnosis and treatment. Mitchell et al. [6] performed a study including $>450$ children to identify which children most need PSG prior to adenotonsillectomy. They showed that information on demographics, physical findings and questionnaire responses does not robustly discriminate different levels of OSAS severity.

\section{Treatment}

Treatment interventions for obstructive SDB are usually implemented in a stepwise fashion starting from the least invasive modality. One or more of the following modalities are usually offered: weight loss; use of anti-inflammatory medications; adenotonsillectomy; orthodontic appliances or rapid maxillary expansion; continuous positive airway pressure (CPAP) or noninvasive positive pressure ventilation; or craniofacial surgery and tracheostomy [14].

The CHAT trial confirmed that early adenotonsillectomy leads to significant improvement in behaviour, quality of life and PSG findings and a significant reduction in symptoms compared to a watchful-waiting group. Normalisation of PSG findings was observed in $79 \%$ of children in the early adenotonsillectomy 
group and in $46 \%$ of the watchful-waiting group. However, the change in attention and executive-function score upon developmental neuropsychological assessment did not differ significantly from baseline to follow-up in both groups. Compared to watchful waiting, surgical treatment of OSAS in school-aged children did not significantly improve attention or executive function, but did reduce symptoms and improved secondary outcomes of behaviour, quality of life and polysomnographic findings, thus providing evidence for beneficial effects of early adenotonsillectomy [15]. Although this study was published prior to this review article's focus, this was the first randomised trial of adenotonsillectomy and papers evaluating different outcomes from the same study were published over the past year.

Rosen et al. [16] evaluated 185 participants (aged 5-9 years with PSG-confirmed OSAS) in the surgical treatment arm of CHAT. Associations were assessed between baseline PSQ or PSG data and baseline morbidity (executive dysfunction, behaviour, quality of life and sleepiness) or postsurgical improvement. They reported that baseline PSG data did not predict these morbidities or their postsurgical improvement. PSQ symptom items, in contrast to PSG results, reflected subjective measures of OSAS-related impairment of behaviour, quality of life and sleepiness and predicted their improvement after adenotonsillectomy. PSQ provided valuable information with respect to behaviour, sleepiness and quality of life and is likely to be useful as an adjunct to PSG in assessment of children with OSAS who may be candidates for surgery.

CHERvin et al. [17] evaluated children with OSAS from the CHAT study who were randomised to the control arm (watchful waiting) and followed-up for 7 months. Nearly half of the children no longer had OSAS on PSG after 7 months of watchful waiting, whereas meaningful improvement in symptoms was not common. Researchers recommended that a baseline low AHI and normal waist circumference, or low PSQ and snoring score, may be helpful to avoid adenotonsillectomy.

Alanso-Álvarez et al. [12] evaluated treatment outcomes of OSAS in a multicentre study of obese children aged 3-14 years recruited from the community. Patients were divided into four groups, as follows. Group 1: non-OSAS with no treatment; group 2: dietary treatment; group 3: surgical treatment; and group 4: CPAP treatment. In obese children with OSAS treated by adenotonsillectomy, there was a high percentage of residual OSAS. Also, in obese children with OSAS treated with nutritional interventions, there was a high percentage of persistent OSAS. The results of the NANOS (Prevalence of Sleep Apnea/Hypopnea Syndrome in Obese Children) trial indicated that obese children have a high rate of persistent disease despite treatment with adenotonsillectomy, nutritional interventions or even nasal CPAP. Furthermore, obese children without OSAS during the initial assessment showed a high risk of developing OSAS at follow-up.

BHATTACHARJEe et al. [18] evaluated serum high-sensitivity C-reactive protein (hsCRP), an inflammatory biomarker, as a predictive factor of residual OSA following adenotonsillectomy. They reported that assessment of post-adenotonsillectomy hsCRP levels compared with the pre-adenotonsillectomy levels emerge as a potentially useful biomarker predicting residual OSA.

\section{Morbidity and long-term outcomes}

SDB can lead to substantial morbidities affecting the central nervous system, the cardiovascular and metabolic systems and somatic growth, ultimately leading to reduced quality of life.

Several studies addressed the cognitive impairment in obese children with OSAS. XANTHOPOULOs et al. [19] performed a study in obese adolescents with OSAS compared to 1) nonsnoring, obese controls without OSAS and 2) nonobese, nonsnoring controls, and reported that obese adolescents with OSAS had significantly worse executive function and attention compared to both obese and lean controls.

VITELL et al. [20] evaluated the impact of obesity on cognitive impairment in children with OSA, children with OSA and obesity, and in normal controls. They also reported that obese children with OSA had higher rates of cognitive impairment.

In contrast to these studies, PIETropaoli et al. [21] demonstrated that SDB of all severities was not associated with cognitive impairment compared to the control group at preschool age. VANDELEUR et al. [22] reviewed the studies evaluating the effect of SDB on cognitive function in preschool children. Although there is enough evidence of impaired cognitive function in school-aged children with SDB, a limited number of studies in preschool children showed that SDB does not have any effect on cognitive function. The authors speculated that the duration of exposure to SDB is important for the difference in findings in different age groups. Cognitive deficits apparent in older children can be due to the cumulative effects on the developing brain of years of SDB. JACKMAN et al. [23] showed that SDB of any severity was associated with poorer behaviour, but not cognitive performance. VANDELEUR et al. [22] asked whether it is more relevant to assess behaviour than cognitive function in younger children, and secondly, whether these deficits in behaviour impact longer term cognitive outcomes. Although there is an absence of long-term longitudinal data, the question arises whether there may be a window of opportunity during which the 
deficits may be prevented if SDB is identified and treated early enough, and perhaps conversely, that treatment instigated beyond this point may be ineffective in improving cognitive outcomes.

Although PSG is the gold-standard diagnostic method for OSA, it is a poor predictor of OSA-associated morbidities. It is important to discover potential biomarkers of OSA-associated morbidities. Of the potentially promising morbidity biomarkers, plasma interleukin-6 and hsCRP appear to exhibit a favourable profile, and may discriminate OSA cases with and without morbidities in both adults and children. Myeloid-related protein (MRP)-8/14 plays an important pathophysiological role in atherosclerosis, and plasma levels correlate with endothelial cell dysfunction. It has been identified as an important predictor of cardiovascular disease. MRP-8/14 was increased in children with obesity and OSA. Considering the physiological importance of neurotransmitters as signalling molecules in the nervous system and the potential alterations that may develop in the context of OSA, urinary neurotransmitters may also provide a good tool for screening OSA related to cognitive morbidity in children [24].

Avis et al. [25] evaluated 8- to 16-year-olds $(n=60)$ with newly diagnosed and untreated OSAS engaged in a virtual reality pedestrian environment. They examined differences in the pedestrian behaviours of looking at traffic, wait time, latency to start road crossing and time to contact by an oncoming vehicle among children with OSAS versus the control group. Children with OSAS were riskier pedestrians than healthy children of the same age, race and sex. Children with OSAS waited for less time before crossing. This study offers initial evidence that OSAS may have significant consequences on children's daytime functioning in a critical domain of personal safety: pedestrian skills.

KaTz et al. [26] evaluated 464 children from the CHAT study. PSG and anthropometry were performed at baseline and 7-month follow-up. An increase in BMI z-score (0.13 versus 0.31$)$ was observed in both the watchful waiting and supportive care (WWSC) and early adenotonsillectomy intervention arms, respectively, but there was a significant increase in the early adenotonsillectomy group. A greater proportion of overweight children randomised to early adenotonsillectomy compared with WWSC developed obesity over the 7 -month interval $(52 \%$ versus $21 \% ; \mathrm{p}<0.05)$. The authors reported that adenotonsillectomy normalises weight in children who have failure to thrive, but also increases risk of obesity in overweight children. Although obese children may benefit from adenotonsillectomy initially, dietary and exercise recommendations should be given following surgery.

OSAS has been associated with cardiometabolic disease in adults. In children, this association is unclear. QUANTE et al. [27] evaluated the effect of early adenotonsillectomy for treatment of OSAS on blood pressure, heart rate, lipids, glucose, insulin and C-reactive protein. There was no significant change in cardiometabolic parameters over the 7-month interval in the early adenotonsillectomy group compared to the WWSC group. Of all measures, overnight heart rate emerged as the most sensitive parameter of paediatric OSAS severity.

KOREN et al. [28] started a trial to evaluate the impact of OSA treatment upon obesity and metabolic outcomes. 69 children with OSA, mean age 5.9 years, underwent baseline overnight PSG, anthropometric and metabolic measurements, adenotonsillectomy and follow-up testing a mean 7.9 months later. 53 children (77\% of the study cohort; $91 \%$ of obese children) had residual OSA (AHI $>1$ event $\cdot \mathrm{h}^{-1}$ ) post-adenotonsillectomy. Fasting plasma insulin (FPI), homeostasis model assessment of insulin resistance and high-density lipoprotein (HDL) improved despite increased BMI z-score. There was no difference between nonobese and obese participants. However, post-adenotonsillectomy BMI z-score, rather than AHI, was the main predictor of levels of follow-up FPI, HDL and other metabolic parameters. This trial showed that adenotonsillectomy improved insulin resistance and HDL, and residual OSA is predicted by baseline FPI and BMI z-score. Adenotonsillectomy cured OSA in $<25 \%$ of all children and only $10 \%$ of obese children; post-adenotonsillectomy PSG is indicated to assess which children still require treatment.

OSAS is a risk factor for cardiovascular adverse events. However, the mechanisms underlying the association are not well defined. Endothelial dysfunction is one of the early markers of cardiovascular complications in OSA. Ultrasonographic assessment of endothelium dependent flow-mediated dilation (FMD) of the brachial artery is the gold standard in assessing endothelial function. FMD is predominantly a result of endothelial nitric oxide release. FMD of the brachial artery correlates well with both coronary endothelial function and the extent of coronary artery atherosclerosis. CHAN et al. [29] compared FMD in children aged 6-18 years with OSA and nonsnoring control subjects and examined its response to treatment. A total of 63 case-control pairs underwent FMD measurement in the morning after overnight PSG. 32 case subjects underwent adenotonsillectomy according to predefined adenotonsillectomy operation criteria. All cases underwent repeat PSG and FMD assessment 6 months later. A significant reduction in obstructive AHI was documented in the adenotonsillectomy group, accompanied by a significant increase in FMD, which was not observed in subjects who did not undergo adenotonsillectomy. They demonstrated that children with OSA had reduced FMD, which was reversible with treatment. 
BIGGs et al. [30] evaluated the long-term effect of treatment of SDB on neurocognition, academic ability and behaviour in a cohort of school-aged children. In this prospective 4-year study, children aged 7-12 years were categorised into treated, untreated and control groups. Children originally diagnosed with SDB and healthy nonsnoring controls underwent repeat PSG and age-standardised neurocognitive and behavioural assessment after 4 years, following initial testing. A decrease in obstructive AHI from time 1 to time 2 was observed in $63 \%$ and $100 \%$ of the untreated and treated groups, respectively. The predictive relationship between change in obstructive AHI and standardised neurocognitive, academic and behavioural scores over time was examined. The trial showed improvements in SDB and in some areas of neurocognition, but there was no difference between treated and untreated groups in terms of academic ability or behaviour.

SPILSBURy et al. [31] studied the incidence, remission and prediction of OSA from middle childhood to late adolescence. The Cleveland Children's Sleep and Health Study participants were recruited and followed for 8 years. 490 participants had overnight PSG data available at ages 8-11 and 16-19 years. OSA prevalence was $\sim 4 \%$ at each examination, but OSA largely did not persist from middle childhood to late adolescence. Habitual snoring and obesity predicted OSA in cross-sectional analyses at each time point. Residence in a disadvantaged neighbourhood, African-American race and premature birth also predicted OSA in middle childhood. Male sex, high BMI, and history of tonsillectomy or adenoidectomy were risk factors among adolescents. Obesity, but not habitual snoring in middle childhood predicted adolescent OSA.

\section{Conclusion}

In conclusion, research in the past year on childhood OSAS taught us that ex-preterm infants are at risk for OSAS and periodic limb movements in later childhood. Although SDB may not be associated with cognitive impairment compared to control subjects at preschool age, schoolchildren with SDB at baseline continue to exhibit significantly poorer behaviour, irrespective of treatment. Children with low SES have decreased access to PSG and treatment. It is possible to perform high-quality home PSGs in children and when inconclusive HRP findings occur, a conventional PSG should be performed. Although objective PSG is needed to diagnose OSAS, symptoms can offer adjunctive insight into comorbidities and surgical response. More research is needed particularly for the diagnosis of mild OSA using HRP in children. Endothelial function is impaired in children with OSA, but reversed with adenotonsillectomy. Adenotonsillectomy normalises weight in children who have failure to thrive, but increases risk for obesity in overweight children. The primary anatomical risk factor for OSAS in obese adolescents is increased size of the pharyngeal lymphoid tissue. Obese children have a high rate of persistent disease despite treatment, and obesity in middle childhood may predict adolescent OSA. Obese adolescents with OSAS have significantly worse executive function and attention compared to both obese and lean controls. OSAS may have significant consequences on children's daytime functioning in a critical domain of personal safety: pedestrian skills.

\section{References}

1 Schwab RJ, Marcus CL, Bagchi S, et al. Understanding the anatomic basis for obstructive sleep apnea syndrome in adolescents. Am J Respir Crit Care Med 2015; 191: 1295-1309.

2 Marcus CL, Meltzer LJ, Roberts RS, et al. Long-term effects of caffeine therapy for apnea of prematurity on sleep at school age. Am J Respir Crit Care Med 2014; 190: 791-799.

3 Tapia IE, Shults J, Doyle LW, et al. Perinatal risk factors associated with the obstructive sleep apnea syndrome in school-aged children born preterm. Sleep 2015 [In press; PMID: 26446117].

4 Marcus CL, Brooks LJ, Draper KA, et al. Diagnosis and management of childhood obstructive sleep apnea syndrome. Pediatrics 2012; 130: e714-e755.

5 Friedman NR, Perkins JN, McNair B, et al. Current practice patterns for sleep-disordered breathing in children. Laryngoscope 2013; 123: 1055-1058.

6 Mitchell RB, Garetz S, Moore RH, et al. The use of clinical parameters to predict obstructive sleep apnea syndrome severity in children: the Childhood Adenotonsillectomy (CHAT) study randomized clinical trial. JAMA Otolaryngol Head Neck Surg 2015; 141: 130-136.

7 Nixon GM, Kermack AS, Davis GM, et al. Planning adenotonsillectomy in children with obstructive sleep apnea: the role of overnight oximetry. Pediatrics 2004; 113: e19-e25.

8 Brouillette RT, Morielli A, Leimanis A, et al. Nocturnal pulse oximetry as an abbreviated testing modality for pediatric obstructive sleep apnea. Pediatrics 2000; 105: 405-412.

9 Kaditis A, Kheirandish-Gozal L, Gozal D. Pediatric OSAS: oximetry can provide answers when polysomnography is not available. Sleep Med Rev 2015; 27: 96-105.

10 Garde A, Dehkordi P, Karlen W, et al. Development of a screening tool for sleep disordered breathing in children using the phone Oximeter ${ }^{\mathrm{TM}}$. PLoS One 2014; 9: e112959.

11 Marcus CL, Traylor J, Biggs SN, et al. Feasibility of comprehensive, unattended ambulatory polysomnography in school-aged children. J Clin Sleep Med 2014; 10: 913-918.

12 Alonso-Âlvarez ML, Terán-Santos J, Navazo-Egüia AI, et al. Treatment outcomes of obstructive sleep apnoea in obese community-dwelling children: the NANOS study. Eur Respir J 2015; 46: 717-727.

13 Boss EF, Benke JR, Tunkel DE, et al. Public insurance and timing of polysomnography and surgical care for children with sleep-disordered breathing. JAMA Otolaryngol Head Neck Surg 2015; 141: 106-111.

14 Kaditis A, Kheirandish-Gozal L, Gozal D. Algorithm for the diagnosis and treatment of pediatric OSA: a proposal of two pediatric sleep centers. Sleep Med 2012; 13: 217-227. 
15 Marcus CL, Moore RH, Rosen CL, et al. A randomized trial of adenotonsillectomy for childhood sleep apnea. N Engl J Med 2013; 368: 2366-2376.

16 Rosen CL, Wang R, Taylor HG, et al. Utility of symptoms to predict treatment outcomes in obstructive sleep apnea syndrome. Pediatrics 2015; 135: e662-e671.

17 Chervin RD, Ellenberg SS, Hou X, et al. Prognosis for spontaneous resolution of OSA in children. Chest 2015; 148: 1204-1213.

18 Bhattacharjee R, Kheirandish-Gozal L, Kaditis AG, et al. C-reactive protein as a potential biomarker of residual obstructive sleep apnea following adenotonsillectomy in children. Sleep 2015 [In press; PMID: 26414898].

19 Xanthopoulos MS, Gallagher PR, Berkowitz RI, et al. Neurobehavioral functioning in adolescents with and without obesity and obstructive sleep apnea. Sleep 2015; 38: 401-410.

20 Vitelli O, Tabarrini A, Miano S, et al. Impact of obesity on cognitive outcome in children with sleep-disordered breathing. Sleep Med 2015; 16: 625-630.

21 Pietropaoli N, Supino MC, Vitelli O, et al. Cognitive function in preschool children with sleep-disordered breathing. Sleep Breath 2015; 19: 1431-1437.

22 Vandeleur M, Widger J. Sleep disordered breathing in preschool children; still smart but for how long? Sleep Breath 2015; 19: 1439-1440.

23 Jackman AR, Biggs SN, Walter LM, et al. Sleep-disordered breathing in preschool children is associated with behavioral, but not cognitive, impairments. Sleep Med 2012; 13: 621-631.

24 De Luca Canto G, Pachêco-Pereira C, Aydinoz S, et al. Biomarkers associated with obstructive sleep apnea and morbidities: a scoping review. Sleep Med 2015; 16: 347-357.

25 Avis KT, Gamble KL, Schwebel DC. Obstructive sleep apnea syndrome increases pedestrian injury risk in children. J Pediatr 2015; 166: 109-114.

26 Katz ES, Moore RH, Rosen CL, et al. Growth after adenotonsillectomy for obstructive sleep apnea: an RCT. Pediatrics 2014; 134: 282-289.

27 Quante M, Wang R, Weng J, et al. The effect of adenotonsillectomy for childhood sleep apnea on cardiometabolic measures. Sleep 2015; 38: 1395-1403.

28 Koren D, Gozal D, Bhattacharjee R, et al. Impact of adenotonsillectomy on insulin resistance and lipoprotein profile in nonobese and obese children. Chest 2015 [In press; DOI: 10.1378/chest.15-1543].

29 Chan KC, Au CT, Chook P, et al. Endothelial function in children with OSA and the effects of adenotonsillectomy. Chest 2015; 147: 132-139.

30 Biggs SN, Vlahandonis A, Anderson V, et al. Long-term changes in neurocognition and behavior following treatment of sleep disordered breathing in school-aged children. Sleep 2014; 37: 77-84

31 Spilsbury JC, Storfer-Isser A, Rosen CL, et al. Remission and incidence of obstructive sleep apnea from middle childhood to late adolescence. Sleep 2015; 38: 23-29. 